Voix et Images

voixetimages

\title{
Les femmes peuvent-elles changer le monde?
}

\section{Lori Saint-Martin}

Volume 21, numéro 2 (62), hiver 1996

Suzanne Jacob

URI : https://id.erudit.org/iderudit/201248ar

DOI : https://doi.org/10.7202/201248ar

Aller au sommaire du numéro

Éditeur(s)

Université du Québec à Montréal

\section{ISSN}

0318-9201 (imprimé)

1705-933X (numérique)

Découvrir la revue

Citer cet article

Saint-Martin, L. (1996). Les femmes peuvent-elles changer le monde? Voix et Images, 21(2), 380-384. https://doi.org/10.7202/201248ar d'utilisation que vous pouvez consulter en ligne.

https://apropos.erudit.org/fr/usagers/politique-dutilisation/ 


\section{Les femmes peuvent-elles changer le monde?}

\section{Lori Saint-Martin, Université du Québec à Montréal}

Il va mal, notre pauvre monde. Chaque jour, le fossé tenace qui sépare les miséreux des nantis s'élargit, tandis que nous assistons, impuissants, à l'effritement de la couche d'ozone, à la montée des intégrismes de tout poil, à l'exacerbation des disparités Nord-Sud. Entre hommes et femmes, les inégalités persistent, malgré l'égalité juridique, toute théorique, que confèrent les chartes et les lois. D'où le changement pourra-t-il bien venir?

Des femmes, peut-être, si seulement on leur en donne l'occasion. Voilà, du moins, ce qu'affirment certaines d'entre elles. Deux livres récents, l'un portant sur l'expérience des femmes en politique fédérale et provinciale ${ }^{1}$, l'autre sur le projet de société issu du "Forum pour un Québec féminin pluriel" de $1992^{2}$, soulèvent des espoirs, des interrogations et, hélas, de graves doutes quant à la capacité réelle des femmes de transformer notre monde, qui en a pourtant bien besoin.

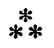

Les femmes, une fois qu'elles auront le pouvoir, arriveront-elles à le mettre au service de la transformation sociale? Pas si sûr. À une époque où une majorité d'hommes comme de femmes politiques se disent favora- 
bles au féminisme, l'expérience des femmes aux trois grandes étapes de la vie politique - l'accès à la scène politique, l'exercice du pouvoir et le départ - n'en continue pas moins d'être marquée par des contraintes particulières. Ce sont ces difficultés que Tremblay et Pelletier s'appliquent à détailler, au moyen d'un questionnaire administré à des parlementaires d'Ottawa et de Québec.

La principale difficulté des politiciennes tient, on s'en doute, au fait qu'elles œuvrent, peu nombreuses, dans un monde pensé par et pour les hommes, où règne une "dictature du masculin" (p. 97). Si l'électorat ne leur est en général pas défavorable, en revanche les instances locales du parti hésitent à les désigner comme candidates, ou encore leur confient des comtés perdus d'avance. La maternité est pour elles un obstacle; elles attendent généralement que leurs enfants aient grandi pour entrer en politique, ce qui n'est pas le cas des hommes. Les stéréotypes de la féminité ont encore cours: on s'attend, disent-elles, à ce qu'elles fassent preuve de minutie et de patience, qu'elles s'intéressent davantage à la santé ou à la culture qu'à l'économie. Surtout, elles sont prises dans une double contrainte: plus elles sont "féminines", moins on les prend au sérieux; inversement, trop "viriles", elles inquiètent, déroutent, font peur. Bref, le monde de la politique, s'il leur est partiellement accessible, demeure hostile.

L'égalité constitue donc pour elles un piège redoutable: elle signifie l'acceptation du masculin comme seule norme et seul critère de valeur, car le discours égalitaire que pratiquent les parlementaires implique que les femmes exercent - ou devraient exercer - le pouvoir politique de la même manière que les hommes. Voilà qui soulève l'épineux problème de la solidarité entre femmes. Si les deux tiers des femmes élues pensent avoir la responsabilité particulière de représenter la population féminine, $60 \%$ de leurs confrères ne leur reconnaissent pas ce rôle, y voyant une forme de discrimination envers les hommes. La fidélité au parti et la nécessité de représenter des intérêts territoriaux entravent encore l'émergence d'une concertation entre femmes politiques de différents partis, de même qu'entre celles-ci et les regroupements féministes (sauf pour de rares questions mobilisatrices comme le droit à l'avortement).

Tremblay et Pelletier appuient la thèse, assez répandue de nos jours, de la "masse critique", à savoir que, lorsque les femmes seront plus nombreuses à accéder à la vie politique (seulement $18 \%$ des députés, à Québec comme à Ottawa, sont des femmes), elles pourront en modifier les règles du jeu. Mais il ne faudra rien de moins qu'une profonde mutation sociale, affirment encore les auteurs: éducation politique véritable proposée aux jeunes femmes comme aux jeunes hommes, assouplissement des rôles selon les sexes, création de nouvelles structures familiales qui évitent aux femmes d'assumer seules la responsabilité de la vie privée. Ce n'est pas demain la veille... Entre-temps, il faudra peut-être compter davantage sur la mobilisation des regroupements féministes que sur 
l'élection de femmes dans les institutions politiques traditionnelles. Autrement dit, le pouvoir n'est pas - ou pas uniquement - là où on le pense.

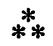

Pour changer le monde. Le Forum pour un Québec féminin pluriel, compte rendu de l'événement et des projets qui en sont issus (rédigé par la journaliste Colette Beauchamp), est un tout petit livre au contenu explosif. On se rappellera que le Forum est né dans la foulée des audiences de la Commission Bélanger-Campeau sur l'avenir constitutionnel du Québec, en 1990, à la suite du refus du pouvoir politique d'accorder un seul siège de commissaire au mouvement des femmes. S'est alors organisée une vaste rencontre regroupant les représentantes de plus de 1000 groupes de femmes. Il en est ressorti un programme national destiné, rien de moins, à "transformer les conditions de vie des femmes québécoises mais aussi celles de l'ensemble de la population" (p. 21). Bref, une nouvelle éthique sociale au féminin. Le projet est totalement, délicieusement, à contre-courant: pour l'engagement massif de l'État dans le secteur des services, contre la mondialisation, l'excellence, l'économisme. À la lecture de cet ouvrage clair, inspiré, tonifiant, on ne sait s'il faut se réjouir de voir tant d'énergie et de bonne volonté déployées ou s'attrister de constater à quel point les femmes continuent à ne pas avoir prise sur le politique. $\mathrm{Si}$, à la différence des féministes de naguère, elles disent rechercher le pouvoir - non pas pour lui-même, mais pour faire advenir un monde nouveau -, si elles prétendent encore transformer le pouvoir lui-même en le faisant passer des institutions politiques traditionnelles aux petits organismes populaires (tables de concertation, syndicats, caisses populaires), force nous est de constater que ce même pouvoir continue en grande partie à leur échapper. Se pose, à la lecture de l'ouvrage, l'éternelle question de la marginalisation ou de la récupération. S'il est quasi impossible d'accéder au pouvoir politique traditionnel sans se faire piéger, il ne reste que la voie, plus marginale, des coalitions, des groupes communautaires, etc., qui œuvrent dans la précarité et le sous-financement chroniques. Si cette voie suscite, chez les participantes, un grand enthousiasme, il ne faut pas oublier pour autant qu'il s'agit de regroupements fragiles, boudés par les grands médias, tenus à l'écart des instances décisionnelles, nécessitant un dévouement de tous les instants qui épuise rapidement leurs militantes.

On ne peut qu'appuyer le projet de société plus humaine, plus généreuse, qui ressort du Forum. Impossible de ne pas souhaiter la réduction des dépenses militaires, l'élimination du gaspillage et de la corruption, l'assainissement de l'environnement. Mais, comme tous les discours à contre-courant, celui-ci risque de ne pas être entendu. À l'ère du désenga- 
gement généralisé de l'État (tendance plus facile à déplorer qu'à contrer), on ne peut guère espérer la mise en œuvre des nombreuses mesures, pourtant fort sensées, que proposent les femmes du Forum, dont la plus importante sans doute est la création d'u un système de sécurité du revenu qui assure réellement à toutes et à tous un montant suffisant pour vivre et s'épanouir" (p. 37).

L'un des mérites de la réflexion issue du Forum, son caractère englobant, fait que le projet de société envisagé est vaste, grisant, mais aussi, peut-être, irréalisable. Des nombreuses idées proposées, beaucoup risquent de demeurer lettre morte. On ne peut légiférer pour faire reconnaître l'égalité des deux sexes dans l'Église, encourager chacun à vivre selon ses besoins réels, sans surconsommation, abolir la compétition et l'exploitation. Il faudrait pour cela une volonté de changement sans pareille, une vague de fond qui emporterait l'ancien monde sur son passage.

Si Tremblay et Pelletier évoquent davantage le conditionnement social que la nature féminine, le discours du Forum, lui, établit, entre les femmes et une série de valeurs dites "féministes" - solidarité, équité, partage, liberté, égalité, respect des différences, pacifisme, antiracisme, antisexisme -, une équivalence stable, viscérale, quasi biologique. Or tous les partis politiques, au Québec, se réclament maintenant de ces valeurs (sans toujours les mettre en pratique). Qu'ont-elles donc de spécifiquement féminin? Il faut se garder d'idéaliser les femmes, en disant par exemple qu'elles "refusent qu'on impose à qui que ce soit une forme de domination" (p. 31). On le sait, chaque fois qu'une féministe occidentale ouvre son ordinateur et se met à rédiger, elle exploite indirectement les travailleuses du Tiers-Monde qui se sont arraché les yeux à l'assembler. On n'échappe pas si facilement au monde dans lequel on vit, et toutes les femmes, même féministes, ne sont pas, hélas, des anges de la concorde.

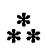

Comme le dit une parlementaire interviewée par Tremblay et Pelletier, "les femmes n'auront jamais été aussi pauvres dans l'histoire de notre société en n'ayant jamais eu autant de droits" (p. 164). À partir de démarches et de présupposés très différents, les deux ouvrages aboutissent à une même conclusion : il ne suffit pas, pour transformer le pouvoir politique, d'élire des femmes. Il faut que des féministes accèdent, nombreuses, au pouvoir. Comment y parvenir? Voilà tout le drame. Les femmes du Forum ont créé une coalition permanente des femmes, un regroupement national qui continuera d'agir. Tremblay et Pelletier prônent, eux aussi, les structures d'échange entre femmes parlementaires et regroupements féministes, telles que les tables de concertation ou les comités mixtes. 
Les revendications que formulent les femmes du Forum, elles le reconnaissent, sont loin d'être toutes nouvelles; l'une des épreuves auxquelles sont confrontées les féministes est précisément la nécessité de se répéter à l'infini, faute de changements concrets. Depuis des années, elles en appellent à la transformation de la culture du pouvoir ou des rapports entre les sexes ${ }^{3}$. Seront-elles entendues un jour? Leurs vœux seront-ils exaucés? On ne prête qu'aux riches, et les femmes sont pauvres. Pauvres en moyens financiers, pauvres en influence. Quémander ou exiger: la différence est ténue lorsque le pouvoir est concentré dans d'autres mains. Si des voix fortes doivent de toute urgence se faire entendre, il est évident que les pouvoirs en place leur feront la sourde oreille. La patience a-t-elle fait son temps, ou demeure-t-elle, malgré tout, la meilleure arme des femmes, celle qui fera advenir, un jour, un monde nouveau?

1. Manon Tremblay et Réjean Pelletier, Que font-elles en politique?, Québec, Presses de l'Université Laval, 1995, $284 \mathrm{p}$.

2. Collectif, Pour changer le monde. Le Forum pour un Québec féminin pluriel, Montréal, les Éditions Écosociété, 1994, 149 p.

3. Voir, par exemple, notre chronique *Du plafond de verre et de la Révolution tranquille ", Voix et Images, vol. XIX, $\mathrm{n}^{\circ} 1$, automne 1993, p. 197-200. 